\title{
Collaborative Optimization of Last Mile Networks for Courier, Express and Parcel Delivery Services
}

\author{
Michael Schwind', Marcel Kunkel2 \\ ${ }^{1}$ Chair of IT-based Logistics, Goethe University Frankfurt \\ 2PickPoint AG, Hofheim-Wallau
}

\section{Introduction}

There is a great deal of pressure to improve delivery effectiveness, especially for the sector of courier, express and parcel services (CEP) networks caused by rising energy costs and fierce competition among carriers. Additionally, society demands higher standards of ecological sustainability management in the transportation business sector (Leonardi and Baumgartner 2004). The fact that the three biggest companies in the world in the logistics sector (UPS, Deutsche Post, FedEx) are CEP service providers illustrates the potential savings of resources that can be achieved by optimizing the delivery networks of these companies (Klaus and Kille 2004). Several thousand vehicles and drivers are used in order to guarantee the in-time delivery of the packages. In many cases last mile delivery is provided by motor carriers which are organized as small independent companies and act as subcontractors of the CEP service providers.

Current optimization approaches for CEP networks mostly rely on the analysis of ex-post data by calculating the optimal location of hubs and depots for the CEP service providers in the network for the long term perspective and by employing tour planning mechanisms for the medium and short term perspective. However, the potential of this kind of ex-post analytics is now nearly completely exhausted. To our knowledge there is no short term ex-ante optimization for the operative handling of last mile delivery planning.

Actual optimization procedures for CEP networks lack the following properties:

- solutions that allow a simulation-based tour planning without predefined fixed delivery areas while simultaneously allowing for the exploitation of driver learning,

- methods that allow the short term exchange of transportation tasks between subcontractors in order to allow a better integration of constraints into the current tour planning methods, 
- applications that enable the reasonable and efficient combination of expost analytics that integrate driver know how by forming basic tour areas (called corridors) with ex-ante optimization including short term tour planning according to the current delivery volume and area structure.

\section{Collaborative Planning in Transportation Networks}

The research and development approach presented here aims at a dynamic optimization of the tour planning process in CEP delivery networks combined with the cooperative exchange of contracts amongst last mile delivery service providers. Our approach touches three major research areas in logistics: the planning of optimal delivery areas in CEP delivery networks, dynamic route optimization including delivery corridors, and the collaborative dispatching of transportation tasks using combinatorial auctions. Only an adequate coupling of these optimization modules will lead to a significant increase in efficiency in CEP delivery networks.

\subsection{Planning of Delivery Areas in Logistic Networks}

CEP solutions that strive for sustainable logistic planning are increasingly focused on the optimization of last mile delivery networks in inner city regions (Fusco et al. 2003, Taniguchi et al. 2003). There is also a challenge to find solutions that will minimize the use of transportation resources for CEP delivery networks in rural areas. However, this type of problem has not been properly investigated due to the fact that the delivery points (DPs) are sparsely distributed in the delivery area (Savelsbergh and Sol 1995). In many cases a two stage process is formulated in order to reduce the complexity of the CEP network optimization problem (Zäpfel and Wasner 2002). The best known approach to find the optimal location of depots in a CEP delivery network is the location routing problem (LRP) which integrates a vehicle routing problem (VRP) to take into account costs of real pickup and delivery tours visiting multiple customers (Nagy and Salhi 2007). However, our focus will lie on the area optimization in the context of the VRP of the last mile delivery process (Daganzo and Erera 1999, Figliozzi 2007, Smilowitz and Daganzo 2007). In order to directly combine the construction of the delivery areas with the tour planning process including driver learning, our CEP optimization approach is designed to be able to deduce the shape of the delivery areas from the iterated calculation of the optimal tours over a time span following the procedure described in section 2.2. Unutilized synergy potentials resulting from overlapping areas will be raised by the use of a combinatorial auction mechanism in a second step (Zäpfel and Wasner 2002, Schwind and Kunkel 2009). 


\subsection{Dynamic Tour Planning with Delivery Corridors}

It can be expected that the adoption of navigation and tour planning systems used in vehicles of the CEP domain will reach the same degree of diffusion as it is the case for the domain of individual transportation. In the sector of field sales there are already a couple of systems available that deal with tour planning and business logic for special applications (Fleischmann et al. 2004, Goel and Gruhn 2006, Cheung et al. 2008). However, this is not the case for CEP delivery networks due to the typical short response and travel time in this sector. The static assignment of vehicles to fixed delivery areas might work well for inner city areas with a high density of DPs. However, this concept shows major weaknesses, especially in rural areas, where the distances between the DPs is large (Powell et al. 2002). In most applications a dynamic assignment of delivery areas is only achieved in the case of capacity shortages caused by demand fluctuations. This is normally done by sending vehicles with spare capacity from one area into neighboring areas. By studying optimal tours generated by solving the vehicle routing problem with time windows ( $V R P T W)$ (Bräysy and Gendreau 2005) one immediately recognizes that the introduction of delivery areas is a factor that can reduce the solution quality because possible overlapping of the tours is inhibited by the borders of the areas. The introduction of delivery corridors that surround the loop-shaped tours, as depicted in Fig. 4, might allow better optimization results. Exact methods are usually not suitable for the optimization problem considered here, because the problem includes many constraints due to the practical requirements (driving times, time windows, etc.) and has to be solved for a large problem size. In order to achieve a satisfying performance, heuristics have to be used. Heuristics that are used to solve this problem are simulated annealing, tabu search ant systems and hybrid methods including constraint propagation (Bräysy and Gendreau 2005).

\subsection{Collaborative Transportation Planning and Combinatorial Exchanges}

One possible way to reduce the complexity of the combined tour and network planning problem for CEP delivery services is to introduce cooperative solutions (Ergun et al. 2007). While doing this, synergy effects between neighboring CEP service providers can be exploited by exchanging delivery tasks and constructing improved tour plans (Schwind et al. 2009). A mechanism that is well suited to achieving this goal is the combinatorial auction (CA) because it can take into consideration the synergies that result from the complementarities that exist between the different sections of a tour (Krajewska and Kopfer 2006). An early approach to introducing the application of CAs in logistic planning was made by Caplice and Sheffi (2003). They combine a route planning process with the allocation of transportation capacity by using a CA that selects the cost-minimal combination of delivery contracts. Elmaghraby and Keskinocak (2005) document a two-step procurement auction for transportation capacities that has been organized by the 
home improvement chain Home Depot to ensure the logistics supply of about thousand stores. Several approaches try to integrate the pickup-and-delivery problem with time windows (PDPTW) to improve usability for real-world transportation problems (Savelsbergh and Sol 1995). Recent approaches address the problem of overlapping delivery areas and indicate cost savings of up to $15 \%$ (Schwind et al. 2009).

\section{Service-oriented Collaborative Transport Optimization}

The use of service oriented architectures (SOA) for the optimization of logistic services has the advantage of the real time integration of different data sources into the optimization. SOA allows one to perform online optimization processes that provide a maximum of flexibility in planning and controlling the delivery process of CEP delivery service providers. Additionally, by introducing simulation scenarios such systems can be used as decision support systems in order to meet short and mid-term decisions such as the cost assessment of a specific delivery structure and allows one vice versa to evaluate the impact of a change in the pricing model on the quality of the entire CEP delivery optimization (Schwind et al. 2007).

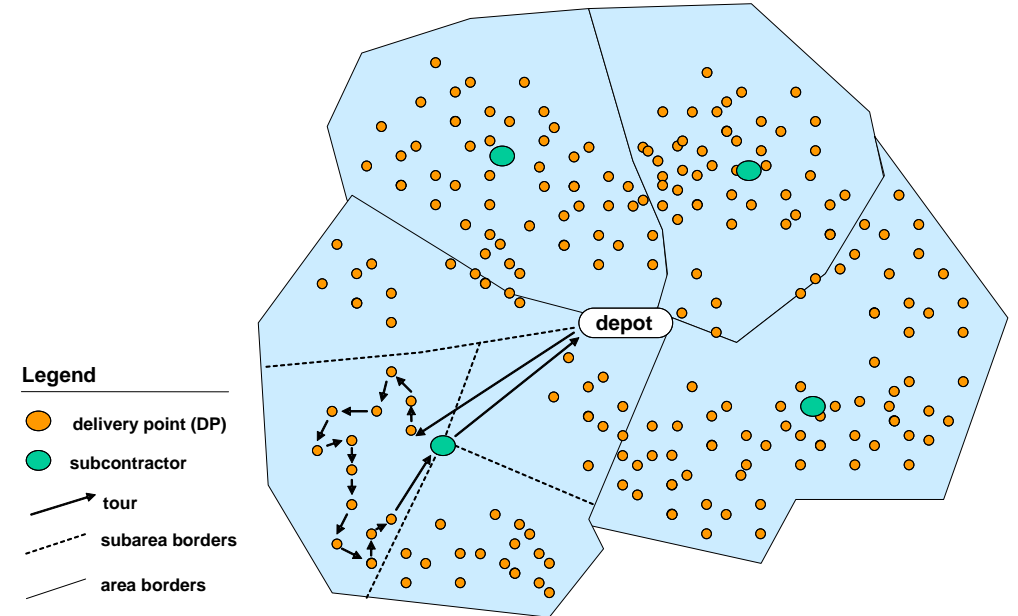

Figure 1: Delivery structure for a ZIP-code-oriented tour planning around a depot

\subsection{Online Optimization of Collaborative Transportation Solutions}

The design of delivery areas in CEP networks currently used follows a multi-stage optimization process due to pragmatic and historical reasons. A classical approach is to follow ZIP code areas to define the main delivery regions and to deploy hubs located in these areas. Additionally, several depots are assigned to the main delivery regions. Within these delivery regions sub-areas are assigned to the depots (Fig. 1) which define the borders of the tours used in the delivery process. The areas are closely related to simple plane structures defined by the ZIP codes and geographi- 
cal circumstances (rivers etc.). Tour planning in conventional CEP delivery allows only tour planning within the borders of such an area for the vehicles of a depot. Additionally this approach implicates long access paths to the delivery areas. An improvement in this situation is provided by the loop system depicted in Fig. 2.

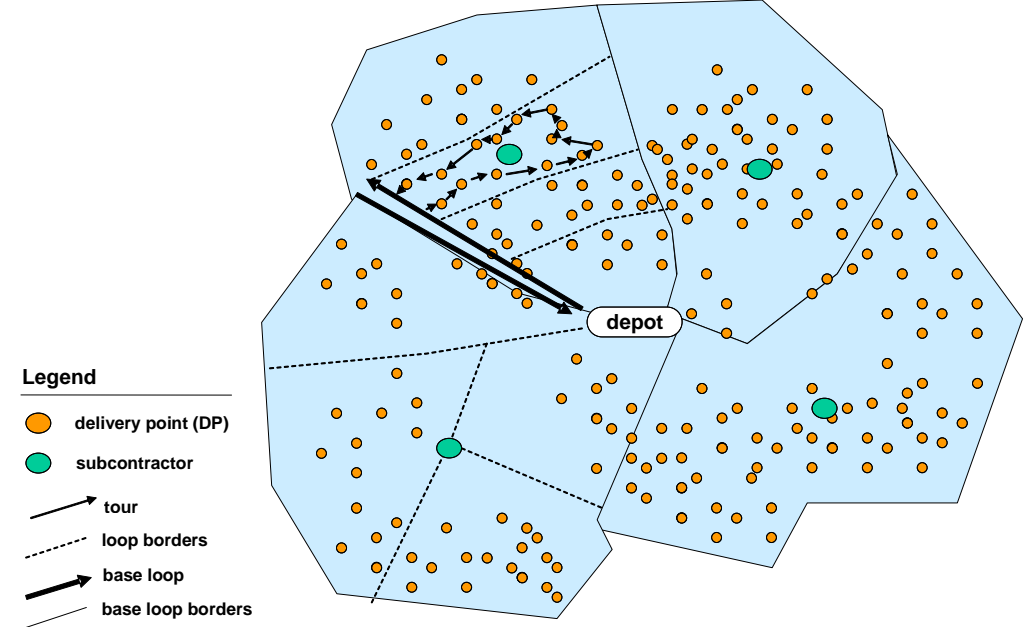

\section{Figure 2: Improved loop-based delivery structure}

Starting from the depots this system defines base loops along characteristic landscapes like rivers and highways. Delivery tours are planned according to the base loops defined. The system allows one to deal with demand fluctuations because the base loop tours themselves are delivery tours feeding sub-tours and the required capacity can be dispatched flexibly by reallocating the load to the sub-tours. Using this system has the additional advantage of providing nearly homogeneous work time for the delivery personnel. However, this base loop system does not provide a further load-dependent ex-ante optimization of the tours on a day-to-day base.

\section{Optimization Approach}

The solution approach presented here should provide a practicable approach for the dynamic routing of packages in a CEP delivery network. This approach has to include constraints and specific factors such as time windows, driver learning in the delivery process etc. while providing a usable result within an appropriate computing time. Fig. 3 presents the proposed solution including three novelties:

1. The system includes an ex-ante tour planning mechanism that permits the formation of master tours in a short computing time and uses a combinatorial auction to find a 'best fit' allocation of 'dispositive DPs' to the tours.

2. The approach provides an efficient way to deal with multiple real-world constraints such as delivery constraints, driving times etc. by introducing 
tour corridors that allow integrate as many DPs as possible into a tour while respecting narrow time windows.

3. The planning model can be extended to include mechanisms that guarantee the incentive compatible allocation of transportation tasks to the subcontractors. By opening the allocation model to external bidders for transportation tasks it is possible to enlarge the proportion of collaborative transportation.

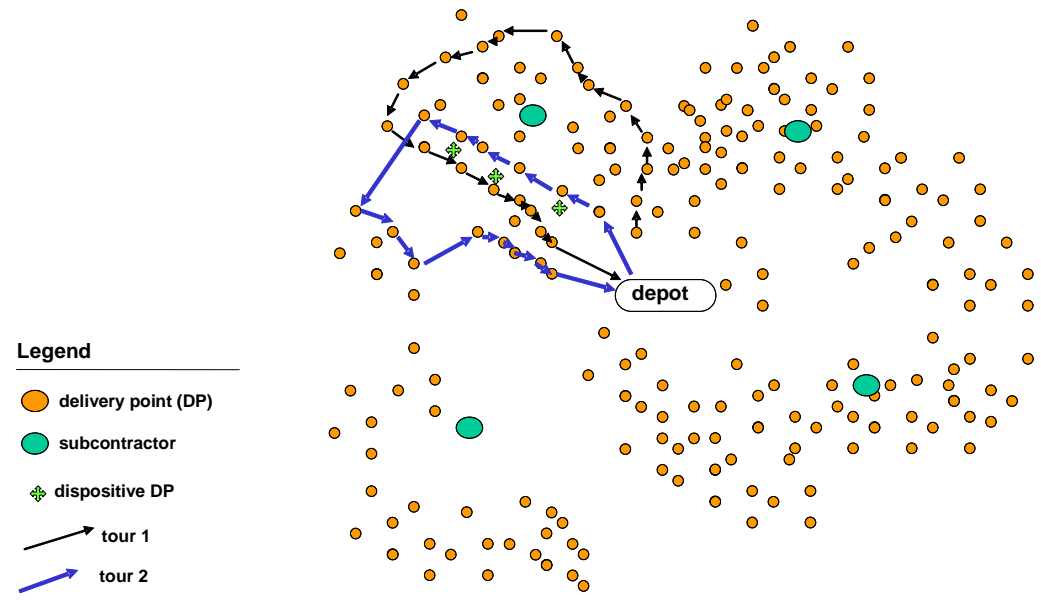

Figure 3: Ex-ante route optimization and the development of master tours

Fig. 3 depicts two delivery tours planned for a day. Frequently visited DPs offer the potential to exploit the learning effect of the drivers with respect to their delivery know how. Crosses (light green) mark dispositive DPs between the master tours that can be assigned to one of the neighbouring tours according to the synergies that result from the allocation. This is achieved by performing a daily combinatorial auction that finds the cost-optimal allocation of DPs to the delivery service providers serving the neighbouring tours. Our approach uses a multi-step process to achieve the optimization goal described above. In a first step optimal tours are calculated on a day-to-day base using historical delivery data. Using a time horizon of 40 to 60 days, the resulting tours are compared to identify similarities and stable patterns that can be used to form the delivery corridors. In order to do this we define corridors as regions in which the DPs can be repeatedly accessed by the same tour without a greater loss of optimization quality. Fig. 4 gives an example depicting two delivery corridors. The corridors include all DPs designated for inclusion into a daily tour. The only task of the tour optimizer is to connect the DPs currently situated in the corridors according to the time window constraints. DPs situated between the corridors are called dispositive DPs (see crosses in Fig. 4) and will be exchanged between the delivery service providers in a second step by using the combinatorial auction in order to achieve a 'best fit' allocation. 


\section{Permanent Optimization of Delivery Corridors with Quality Assessment}

By using the delivery corridor approach we can reduce the ex-ante complexity and simultaneously allow the use of human knowledge and driver learning respectively. However, the multi-step approach reduces the quality of the optimization process. For this reason it is important to find a trade-off between the positive effects of driver learning and the quality reducing impact of the corridor formation mechanism. In order to quantify this impact it seems to be valuable to compare the current result with a reference solution based on global optimization. By doing this in simultaneous simulations the optimal parameterization of the solution method should be found. By using simulations we can determine the optimal shape of the delivery corridors according to the global optimization goal and the specific transportation task under constraints. Broader corridors and with this a lower number of dispositive DPs lead to the increased stability of the tour planning process. By contrast, smaller corridors lead to a higher number of dispositive DPs causing higher flexibility of the tour planning process and should lead to an optimization result closer to the global optimum. This, however, will reduce the positive impact of the diver learning process. The daily monitoring of the flexibility / learning trade-off should provide the optimal parameterization and performance of the entire system. The deviation of the current tour planning including the corridor solution from the optimal global solution can be used as quality function to measure the system's performance.
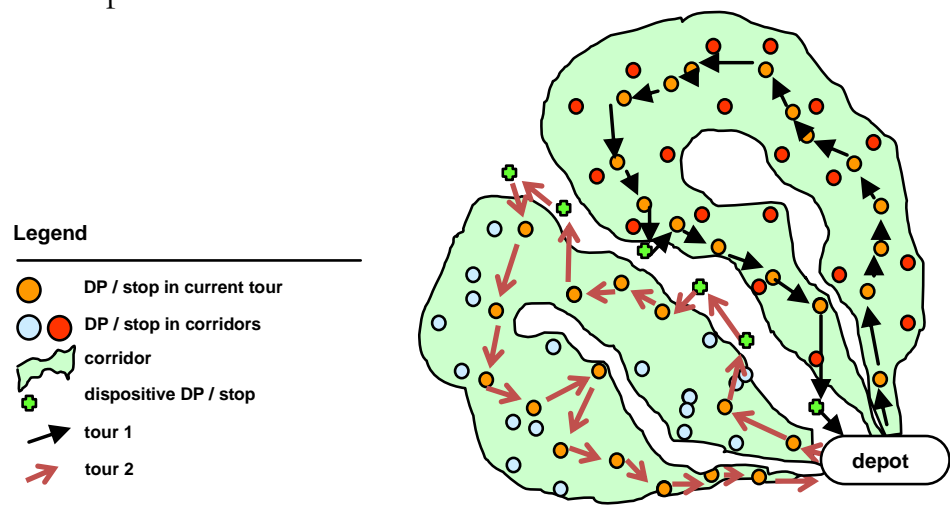

Figure 4: Formation of delivery corridors in the master tour optimization process

In the following section we will describe the change of the master tours measured on a daily base as drift. The drift can also serve as a measure for the reproducibility of the current solution. Factors militating against good solution quality are a high level of constraints (especially time window constraints) and a high level of heterogeneity in the daily demand profiles of the DPs. One can imagine in this context that a very short delivery time window for a certain stop (DP) would lead to a very low probability that this DP can be efficiently integrated into a stable corridor model. In contrast to this, human-driven constraints may be 
counterproductive with respect to the flexibility of the solution if learning impact is overestimated and the driver is stuck in a certain tour profile. This is especially the case for tours that have complex shapes and do not fit into predefined corridors. The concept of drift is depicted in Fig. 5 showing the gradual shift of the corridors in the course of time for every day-to-day aspect. The calibration of the drift parameters is an essential success factor in our model. Crucial parameters might include the number and the type of dispositive DPs per drift on a daily base. The use of drift in the master tour building process should have 3 advantages:

1. drivers are able to learn the characteristic of new environments stepwise

2. smooth transition without sharp structural changes

3. well distributed use of computational resources due to ex-post calculation of drift (after the daily tour optimization process)

In order to find a suitable parameterization of the drift it is necessary to additionally regard the behaviour of the human actors in the CEP delivery process. It is therefore important to introduce incentives into the delivery process that motivate the actors to describe their specific know how (constraints, relaxations), so that it can be integrated into the software solution.

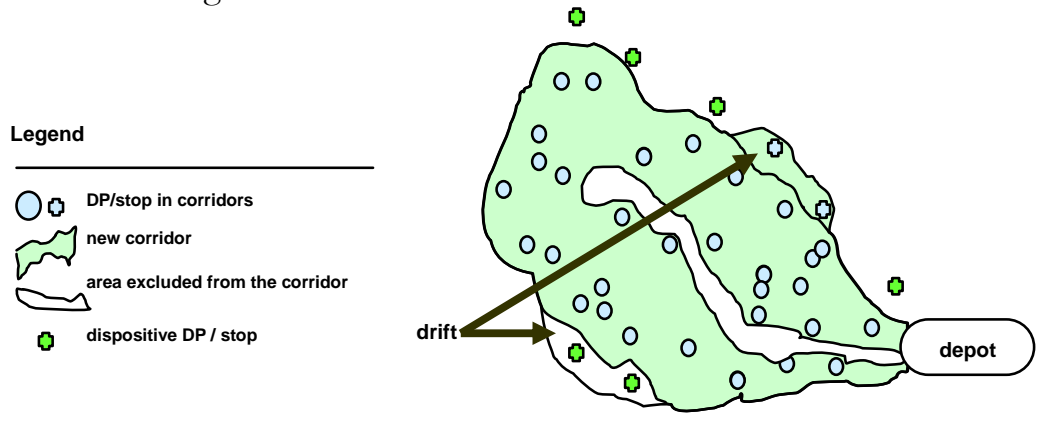

Figure 5: Drift with master tour formation

In the beginning of the 80 s the CEP service provider UPS had already integrated such information into the delivery planning process by performing REFA time studies. These studies analyzed and optimized the behaviour of the personnel in a guided procedure to improve the overall performance of the delivery process. This process emphasized the learning process of the personnel and was able to integrate their local know how into the delivery process by setting up easy rules, e.g. for a delivery on the first floor only use the staircase, avoid elevator or only turn right at traffic lights. A recent approach that integrates this learning concept into a modern IT-supported variant is presented by Zhong et al. (2007).

\section{Combinatorial Auction Mechanism for the Exchange of Delivery Contracts}

As already mentioned in section 3.1, there are dispositive DPs that can be assigned to one delivery corridor or another in the optimization process. In order to do this 
we use combinatorial auctions with a cost valuation of DPs based on their specific properties such as geographical location, delivery time windows, etc. to exchange transportation tasks between the sub-contractors in the CEP last mile delivery network of the CEP service providers.

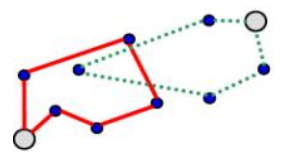

a)

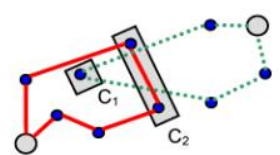

b)

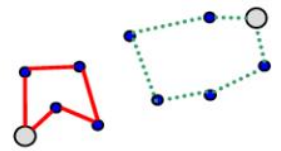

c)

Figure 6: Exchange mechanism of the combinatorial auction

Fig. 6a depicts the initial situation of two tours with DPs already assigned. The DPs that are located in the overlapping areas of the tours are designated for exchange and are arranged in the form of clusters according to their geographical location and the delivery time windows in order to be able to exploit the synergies resulting between the different DPs. (Fig. $6 \mathrm{~b}$, cluster $\mathrm{C}_{1}$, comprising one DP only and cluster $\mathrm{C}_{2}$, containing two DPs). The cost savings that are achievable for the outsourcing of a DP with a neighbouring delivery service provider are calculated for each cluster. Similarly the delivery service providers calculate the cost for the insourcing of released clusters into their tour. These costs are used as bid prices in the combinatorial auction mechanism. As a result, the auction is able to find the optimal allocation of the dispositive DPs to delivery service providers. Finally the system calculates the delivery tours based on this allocation (Fig. 6c).

\section{A SOA-based Decision Support System for CEP Logistics Management}

The KolOptNet system described here might also be used to improve the entire CEP delivery service network because all relevant data and impact factors are known a priori and can be used to perform a global network optimization. The model can be extended to provide decision support for the operators of a CEP delivery network by analyzing the impact of inclusion and exclusion of DPs or a change in the delivery structure on the system's economic status (by calculating parameters such as e.g. marginal cost, optimal pricing, risk profile). Using a similar systematic that allows the users can evaluate other forms of objective functions with respect to the service level achievable for the CEP delivery process in the service network.

Given a certain service level that should be reached with a certain degree of confidence the KolOptNet system is able to analyze the effect of a change in parameters in terms of monetary units added or lost for the financial yield. For example, if a CEP delivery service provider that promises a 99.5\% confidence for the $10^{00}$ delivery of a package wants to know how much the cost reduction produced by lowering the service level to $99 \%$ would be, the decision support of the $K o$ lOptNet system can provide an answer by performing a simulation. It is also possi- 
ble to generate product variation by guaranteeing different service levels for the DPs. Such products have pricing differentiated according to their production costs.

\subsection{A Service Oriented Architecture for Collaborative Transport Optimization}

A major goal of the KolOptNet project is to develop a software prototype that uses the existing IT-infrastructure of PickPoint AG to evaluate the innovative combined route planning and combinatorial auction infrastructure ${ }^{1}$. The basic element of the system architecture is the tour planning service that has access to the MapPointbased distance calculation service, the drift service, the Combinatorial Exchange (ComEx) service (Schwind et al. 2009), and the tour optimizer. Additionally, the entire system requires the external services depicted in Fig. 7. The system makes use of static master data such as delivery area information and dynamic temporal data like the current movement of the vehicles and packages. The major output data of the entire system is sorting plans for the packages and tour plans for the vehicles. This data is transmitted to the wireless mobile package delivery scanner devices (WMPDSDs) that interactively control the delivery process. Additionally these WMPDSDs are used to permanently collect data from the generic delivery process (scanning of packages, customer's signature, proof of delivery, proof of collection, time stamp. etc.). The integrated GUI-based report systems allow one to manage and control the entire system and provide many intervention options.

A central engine, the tour planning service, uses these services for daily tour planning. The service relies on master data (regional information, geography of the area in order to analyze the sub-contractors with the service level agreements (SLAs)) and provides the underlying data for the packages (delivery address, size information, SLAs). In order to accomplish the daily distribution the following steps have to be performed:

1. preprocessing of package information (clean geo coding) and daily assignment of DPs to master tours, request for optimal corridors from the tour planning service including drift service information

2. calculation of the optimal master tours using the tour optimizer that uses a pre-calculated distance matrix in order to calculate the cost of the tour segments that have to be arranged in the right sequence

3. identification of dispositive DPs outside the corridors and clustering into route bundles by the tour planning service using heuristics (e.g. nearest neighbor) and allocation of these bundles using the ComEx auction service

4. calculation of the preliminary tours and data preprocessing for use with the mobile tour planning service

${ }^{1}$ www.koloptnet.de, www.pickpoint.de 
5. tour planning service calculates the sequence in which the packages should be loaded on the vehicles and dispatches this information to the depots' sorting stations

6. provision of tour information (sequence of stops per tour) for the database server and dispatch to the scanner server and PickPoint web server for the graphical visualization of tours

7. scanner server dispatches the tour information and package data to the WMPDSDs

8. loading and sorting of the packages with a scanning process at the depots

9. initialization of the tour planning service supported by the WMPDSDs including feedback of current delivery status and actual DP information (stop time, driving time)

10. WMPDSDs record and report the data of the current tours to the scanner servers which archive the delivery data that is used to calculate the optimal corridors and their drift for the following day

11. calculation of appropriate drift parameters for the following day using actual and historical data for a predefined time window
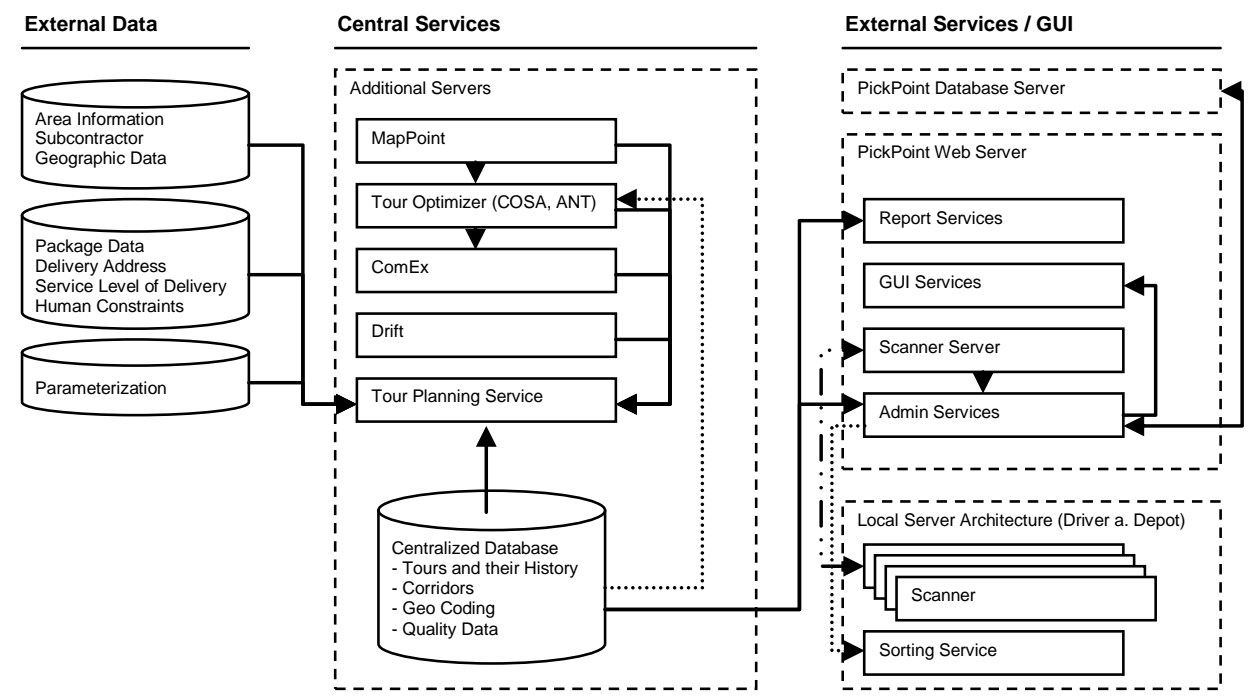

Figure 7: Service architecture of the KolOptNet system

\section{Conclusion}

The service-orientated software system KolOptNet described here is designed to improve the efficiency of CEP delivery service networks. Our approach allows the optimization of last mile tours for a CEP delivery network on a day-to-day basis by introducing combined planning heuristics. Additionally, combinatorial auctions are 
used to enable the flexible exchange of DPs between the service providers that often operate as autonomous sub-contractors in the CEP delivery networks. Our KolOptNet software provides a SOA-orientated decision support system that analyzes historical data based on ex-post simulations in order to guarantee the optimal cooperation of the various optimization systems introduced into the delivery planning process. In a second step we use ex-ante simulations to achieve improved planning for the last mile delivery process. The entire system should be able to find a suitable parameterization that achieves the maximum cost savings. By iteratively integrating ex-post simulation and ex-ante planning steps into the KolOptNet system we can assess the learning effect of the human actors into the optimization of the delivery process. In order to do this the system is introduced in a real world application at a major French CEP delivery service provider.

\section{References}

Bräysy O, Gendreau M (2005): Vehicle Routing Problem with Time Windows, Part I: Route Construction and Local Search Algorithms. In: Transport. Sci. 39(1):104-118.

Caplice C, Sheffi Y (2003): Optimization-based Procurement for Transportation Services. In: J Bus Logist 24(2):109-128.

Cheung BKS, Choy KL, Li C-L, Shi W, Tang J (2008): Dynamic routing model and solution methods for fleet management with mobile technologies. In: Int. J. Prod. Econ. 113, p. 694-705.

Daganzo CF, Erera A (1999): On Planning and Design of Logistics Systems for Uncertain Environments. In: Speranza G, Stahly P (Eds.): New Trends in Distribution Logistics. Springer, p. 100-105.

Elmaghraby W, Keskinocak P (2000): Technology for Transportation Bidding at 'The Home Depot'. School of Industrial and Systems Engineering, Georgia Institute of Technology.

Ergun O, Kuyzu G, Savelsbergh MWP (2007): Shipper Collaboration. In: Comp. \& Oper. Res. 34(6):1551-1560.

Fleischmann B, Gnutzmann S, Sandvoß E (2004): Dynamic Vehicle Routing Based on Online Traffic Information. In: Transport. Sci. 38(4):420-433.

Fusco G, Tatarelli L, Valentini MP (2003): Last-mile, a Procedure to set up an Optimized Delivery Scheme. In: Delivering the Goods: 21st Century Challenges to Urban Goods Transport. OECD Publishing, p. 147-161. 
Goel A, Gruhn V (2006): A fleet monitoring system for advanced tracking of commercial vehicles (Eds.): Proceedings of the IEEE Int. Conference on Systems, Man, and Cybernetics 2006, Vol. 1-6, IEEE, NY, p. 2517-2522.

Krajewska MA, Kopfer H (2006): Collaborating Freight Forwarding Enterprises. In: OR Spectrum 28(3):301-436.

Klaus P, Kille C (2006): Die Top 100 der Logistik: Marktgrößen, Marktsegmente und Marktführer in der Logistikdienstleistungswirtschaft. In: Studie des Fraunhofer IIS Nordostpark 93, Nürnberg.

Leonardi J, Baumgartner M (2004): $\mathrm{CO}_{2}$ Efficiency in Road Freight

Transportation: Status quo, measures and potential. In: Transport. Res. D-Tr. E. 9(6):451-464.

Nagy G, Salhi S (2007): Location-Routing: Issues, Models, and Methods. In: Eur. J. Oper. Res. 177, p. 649-672.

Powell B, Marar A, Gelfand J, Bowers S (2002): Implementing Real-Time Optimization Models: A Case Application from the Motor Carrier Industry. In: Oper. Res. 50(4):571-581.

Savelsbergh MWP, Sol M (1995): The General Pickup and Delivery Problem. In: Transport. Sci. 29, p. 17-29.

Schwind M, Hinz O, Stockheim T, Bernhardt M (2007): Standardizing Interactive Pricing for Electronic Business. In: Electronic Markets 18(2):161-174.

Schwind M, Gujo O, Vykoukal J (2009): A Combinatorial Intra-Enterprise Exchange for Logistics Services. In: Information Systems and eBusiness Management 7, p. 447-471.

Smilowitz K, Daganzo CF (2007): Continuum Approximation Techniques for the Design of Integrated Package Distribution Systems. In: Networks 50(3):183196.

Taniguchi E, Thompson RG, Yamada T (2003): Visions for Cities Logistics. In: Delivering the Goods: 21st Century Challenges to Urban Goods Transport. OECD Publishing, p. 1-16.

Zäpfel G, Wasner M (2002): Planning and optimization of hub-and-spoke transportation networks of cooperative third-party logistics providers. In: Int. J. Prod. Econ. 78(2):207-220.

Zhong H, Hall RW, Dessouky M (2007): Territory Planning and Vehicle Dispatching with Driver Learning. In: Transport. Sci. 41(1):74-89. 